\title{
Correlation of serum microRNA-122 level with the levels of Alanine aminotransferase and HBV-DNA in Chronic HBV-infected patients
}

\author{
Rana Tabar Asad Laleh ${ }^{1}$, Zohreh Sharifi ${ }^{1} * \mathbb{D}$, Ali Akbar Pourfathollah ${ }^{1,2}$ \\ Received: 6 Jul 2019 \\ Published: 19 Oct 2021
}

\section{Abstract}

Background: The microRNA-122 (miR-122) is a liver-specific microRNA that can be used as a potential molecular marker for predicting liver injury. There is a positive correlation between miR-122 level in serum and hepatitis B virus (HBV) replication in patients infected with this virus. The present study was conducted to study the clinical importance and expression of miR-122 in asymptomatic and symptomatic patients with HBV infection in comparison to the healthy group.

Methods: This study was performed on 60 samples to examine the presence of HBsAg and total HBc antibody (IgM-IgG) using an enzyme-linked immunosorbent assay. HBV-DNA extraction and real time polymerase chain reaction (PCR) assay were performed on all samples via the Real ART HBV LC PCR kit on a LightCycler instrument. RNA was extracted from the serum of all participants. Next, miRNA expression was assessed using quantitative real time reverse-transcription PCR. Also, ALT levels were measured as a surrogate parameter for liver injury using Pars Azmoon Biochemical assay Kit on Hitachi autoanalyzer. The Levene , Kruskal Wallis, Mann-Whitney and Spearman's correlation tests were used for assessing the differences between the studied groups.

Results: Based on the obtained results, miR-122 expression in patients with HBV without clinical symptoms was 1.6 times, while in patients with clinical symptoms was 2.7 times more than the control group $(p=0.001)$. A significant increase was observed in the ALT enzyme of symptomatic patients $(\mathrm{p}=0.001)$. HBV DNA in the people with clinical symptoms was higher than $105 \mathrm{copies} / \mathrm{mL}$ and in the asymptomatic group was less than 103 copies $/ \mathrm{mL}$, suggesting a statistically significant increase in a group with clinical symptoms $(\mathrm{p}=0.001)$. Finally, it was found that the miR-122 serum concentration correlated with HBV DNA and serum ALT ( $\mathrm{p}=0.001)$.

Conclusion: Based on the obtained results, measuring the miR-122 levels can serve as a biomarker and an indicator of hepatitis B replication, especially in cases where ALT levels are unchanged; however, more research and more samples are needed.

Keywords: MicroRNA, Hepatitis B, MiR-122, ALT

Conflicts of Interest: None declared

Funding: This study has been performed with the financial support of the High Institute for Research and Education in Transfusion Medicine, Blood Transfusion Research Center.

*This work has been published under CC BY-NC-SA 1.0 license.

Copyright $\mathbb{C}$ Iran University of Medical Sciences

Cite this article as: Tabar Asad Laleh R, Sharifi Z, Pourfathollah AA. Correlation of serum microRNA-122 level with the levels of Alanine aminotransferase and HBV-DNA in Chronic HBV-infected patients. Med J Islam Repub Iran. 2021 (19 Oct);35:137. https://doi.org/10.47176/mjiri.35.137

\section{Introduction}

Hepatitis B virus (HBV) is the most prevalent infectious disease worldwide (1). Eradication of the infection by this hepatotropic noncytopathic DNA virus is still a global health concern. About 257 million people in the world carry HBV as persistent hosts and many of them become

Corresponding author: Dr Zohreh Sharifi, z.sharifi@tmi.ac.ir

1. Blood Transfusion Research Center, High Institute for Research and Education in Transfusion Medicine, Tehran, Iran

2. Departments of Immunology, Faculty of Medical Sciences, Tarbiat Modares University, Tehran, Iran chronic liver patients. Every year, 1 out of each 2 million people die due to illnesses related to $\mathrm{HBV}(2)$. In the majority of adults, infection of HBV is self-restricting and a fast clearance of virus occurs, while in some other cases, the infected people become only carriers or survive with a

$\uparrow$ What is "already known” in this topic:

The microRNA-122 is a hepatocyte-specific microRNA that can be used as a potential molecular marker for predicting liver injury.

\section{$\rightarrow$ What this article adds:}

Measuring the miR-122 levels can serve as an indicator of hepatitis B replication, especially in cases where ALT (alanine aminotransferase) levels are unchanged. 
chronic persistent $\mathrm{HBV}$ infection.

Based on the serologic profiles of HBV, the infected individuals (3) can be classified into 2 groups: (1) asymptomatic HBV carriers (ASCs) and (2) individuals with chronic hepatitis B (CHB). ASCs, present a low viral load due to long-term viral replication inhibition. (ie, $<2,000$ $\mathrm{IU} / \mathrm{mL}$ ). In addition, they do not present any ultrasonography, biochemical, and histological sign of liver injury (4).

MicroRNAs are a large family of small noncoding RNAs of about 22 nucleotide length that regulate gene expression through base-pairing with the end of 3'mRNA target (3). These mRNAs have a significant role in differentiation, cell development, and physiological function. Moreover, they control the progression of viral infections, tumors, and other diseases similar to $\operatorname{HBV}(5)$. These viruses can affect the microRNA expression profiles positively to promote replication potential and pathogenesis (6).

It has been evidenced that circulating miRNAs are among the potential molecular markers that may lead to CHB-induced liver injury $(7,8)$. The level of liver damage and the immune characteristics are within a range of ASCs to CHBs. In this regard, also the expression of miRNAs profiles is different between the groups (ASCs and CHBs) of HBV-infected individuals (3).

Alanine aminotransferase (ALT) and aspartate aminotransferase in blood samples are among the most commonly used enzymatic indicators for evaluating liver function. However, a liver function test result does not always represent liver disease. On the other hand, liver cells damage and ALT release in HBV infection are induced by an immune response to HBV and not the virus (6).

In this study, the levels of miR-122 and the ALT enzyme were measured and compared in 3 groups; that is, 2 groups of patients who were asymptomatic and symptomatic HBV-infected patients and healthy participants as the control group.

\section{Methods}

\section{Experimental design}

The participants of this cross-sectional study included 60 participants (52 men and 8 women), of whom 20 were blood donors with asymptomatic hepatitis B infection, 20 were chronic hepatitis B (CHB), and 20 were healthy individuals as the control group. All patients coinfected with $\mathrm{HCV}$ or HIV; also, those with other liver diseases, such as autoimmune hepatitis, alcohol consumption, or under treatment with hepatotoxic drugs were excluded from the study. The present research was approved in Ethics Committee of High Institute for Research and Education in Transfusion Medicine (Number: IR.TMI.REC.1396.013). Also, before the study, all participants willingly signed written informed consent.

\section{Serological tests}

All serum samples were stored at a temperature of -80 ${ }^{\circ} \mathrm{C}$. Moreover, all 60 participants were tested for HBs-Ag (Siemens), based on the manufacturer's instructions. The serum samples were tested using the enzyme-linked immunosorbent assay for total anti-HBc Ab (IgM-IgG) (Di-
aPro) based on the instructions of the manufacturer. Eventually, the reactive anti-HBc results were rechecked. The correlation strength between serum microRNA-122 level with the levels of ALT and HBV-DNA was analyzed by odd ratio.

\section{Real-time PCR}

For detection of viral load in the collected samples, viral-DNA was purified using the QIAamp DSP Virus Kit (Hilden) based on the instructions of the manufacturer. Afterward, to validate inhibition in PCR reaction and DNA extraction, real time polymerase chain reaction assay was performed on all samples through Real ART HBV LC PCR kit on a LightCycler system.

Also, RNA extraction was done using $2 \mathrm{~mL}$ whole blood by miRNA Isolation Kit, miRNeasy Mini Kit QIAGEN, following the manufacturer guidelines. To evaluate the miRNA expression, a semi-quantitative real-time PCR was done using Pars genome, Iran mir Amp kit.

Small nuclear RNA U6 (RNU6) was used as a reference gene. Also, serial dilutions of cDNAs were used for drawing standard curves (the miR-122 and RNU6) to evaluate the efficiency of real time PCR reaction. The real time PCR (with a coefficient of determination $\left[\mathrm{R}^{2}\right]$ equal to 0.998 and the curve slope of -3.358) was performed in triplicate according to the manufacturer's instruction under these conditions: $95{ }^{\circ} \mathrm{C}$ for 10 minutes, followed by 40 cycles of $95^{\circ} \mathrm{C}$ for 5 seconds, $61^{\circ} \mathrm{C}$ for 20 seconds, $72^{\circ} \mathrm{C}$ for 20 seconds, and a melt curve analysis by heating from $50^{\circ} \mathrm{C}$ to $95^{\circ} \mathrm{C}$, with a rate of $0.1^{\circ} \mathrm{C} / \mathrm{s}$. Finally, an ALT level was measured as a surrogate parameter for liver injury using Pars Azmoon Biochemical Assay Kit in a Hitachi autoanalyzer.

\section{Statistical analysis}

The collected data were analyzed using the $2-{ }^{\Delta \Delta C t}$ method. The fold-change was calculated by the difference in the means of the miR-122 Ct- RNU6 Ct based on the 2$\Delta \Delta \mathrm{Ct}$ method. SPSS statistical software Version 22.0 (SPSS Inc) was used for the data analysis, with $\mathrm{P}<.05$ considered as the significance level. All results were reported as means $\pm \mathrm{SD}$. The Levene, Kruskal Wallis, and Mann-Whitney tests were used for assessing the differences between the studied groups. The Spearman's correlation was used for the correlation analysis of the collected data. The Correlation strength between serum microRNA122 level with the levels of ALT and HBV-DNA was analyzed by odd ratio.

\section{Results}

As can be seen in Table 1, the mean age in the asymptomatic group was $39.3 \pm 6.9$ years, which is more than the mean age of the control group $(37.3 \pm 6.5$ years $)$ and the group with clinical symptoms ( $38 \pm 8.14$ years). The value of the HBV DNA variable in the group with clinical symptoms was $>10^{5}$ copies $/ \mathrm{mL}$, while in the asymptomatic group was $<10^{3}$ copies $/ \mathrm{mL}$. Also, the descriptive findings in Table 1 show that the mean of ALT in the clinical symptoms $(85.5 \pm 18.5 \mathrm{U} / \mathrm{L})$ was greater than that of the asymptomatic group $(19.23 \pm 9 \mathrm{U} / \mathrm{L})$, while in the control 
Table 1. Descriptive and Serological Characteristics of the Participants in 3 the Studied Groups

\begin{tabular}{|c|c|c|c|c|c|c|c|}
\hline Group & $\begin{array}{c}\text { Age mean } \\
\text { (years) }\end{array}$ & $\begin{array}{l}\mathrm{HBsAg}>6 \\
\text { months }\end{array}$ & Anti-HBc & $\begin{array}{l}\text { Viral load median } \\
\quad(\text { copies } / \mathrm{ml})\end{array}$ & $\begin{array}{l}\text { ALT median } \\
(\mathrm{U} / \mathrm{L})\end{array}$ & $\begin{array}{l}\text { MiR-122 fold- } \\
\text { change }\end{array}$ & *P value \\
\hline Control & $37.3 \pm 6.5$ & - & - & - & $16.25 \pm 5.6$ & - & - \\
\hline Asymptomatic & $39.3 \pm 6.9$ & + & + & $7.20 \times 10^{3} \pm 162$ & $19.23 \pm 9$ & $1.57 \pm 0.05$ & 0.013 \\
\hline Symptomatic & $38 \pm 8.14$ & + & + & $2.74 \times 10^{5} \pm 548$ & $85.5 \pm 18.5$ & $2.75 \pm 0.08$ & 0.011 \\
\hline
\end{tabular}

group (16.25 $\pm 5.6 \mathrm{U} / \mathrm{L})$ it was less than the other 2 groups. Also, it is observed that the mean of the viral load median in the clinical symptoms $(2.74 \times 105 \pm 548$ copies $/ \mathrm{mL})$ was greater than that of the asymptomatic group $(7.20 \times 103 \pm 162$ copies $/ \mathrm{mL})$. The increased expression levels of miR-122 in CHB and AHB was observed with the odds ratio of 1.75 and a $95 \%$ CI of 1.67 to 1.83 and the odds ratio of 0.7 , and a $95 \% \mathrm{CI}$ of 0.67 to 0.73 , with a cutoff value of 0.71 and 0.57 , respectively.

The results of the Mann-Whitney test were employed to compare the fold-change of the miR-122 variable between each pair of groups. Significance level in the first comparison at a 99\% CI showed that the control group had a statistically significant difference with the asymptomatic group $(\mathrm{p}=0.013)$.

A significant level of preparedness at $99 \%$ in the second comparison of the level suggested the presence of a statistically significant difference between the control group and the clinical symptoms $(\mathrm{p}=0.011)$. Also, a significant level of preparedness at $99 \%$ in the third comparison showed that the asymptomatic group was significantly different from the clinical symptoms $(p=0.001)$. Comparing the ALT variable among the 3 control, asymptomatic, and symptomatic groups revealed the significant level of preparedness in the first comparison at 95\% CI. However, the control group was not significant with the asymptomatic group $(\mathrm{p}=0.150)$.

The significance level obtained in the second comparison showed that at $99 \%$ level of confidence, there was a significant difference between the control and symptomatic groups statistically $(\mathrm{p}=0.011)$. A significant level of preparedness in the third comparison showed that $99 \%$ of the asymptomatic group had a significant difference with the clinical symptoms ( $\mathrm{p}=0.013$ ). Also, the Mann-Whitney test was applied to compare the HBV viral load variable between the asymptomatic group and clinical symptoms group. A significant level of preparedness in the comparison revealed that $99 \%$ of the asymptomatic group had a significant difference with the clinical symptoms $(\mathrm{p}=0.001)$, and the HBV viral load variable in the asymptomatic group was lower than that in the group with clinical symptoms.

The Spearman correlation coefficient was applied to measure the relationship between the fold-change of miR122 and ALT (Fig. 1), by which a direct relationship was found between them $(p=0.001, r=0.89)$.

The Spearman correlation coefficient was applied for investigating the relationship between fold changes of miR-122 with HBV viral load (Fig. 2). The findings revealed a positive correlation between these 2 variables $(\mathrm{p}=0.001, \mathrm{r}=0.92)$.

\section{Discussion}

Chronic HBV infection is a factor that may change the expression of a miRNA molecule. This infection can particularly alter the liver-specific expression patterns of miRNA. These changes can lead to disease progression and persistent chronic infection. Elucidating these variations is of great assistance in explaining the CHB infection mechanism. On the other hand, it develops new markers for molecular diagnosis and evaluation of the efficiency of antiviral treatments.

In this study, 2 groups of patients (ie, asymptomatic and chronic HBV infected) showed an increase in miR-122 levels. The most popular miRNAs are found in the liver and are of high importance in determining the biological and clinical functions. According to the report of re-

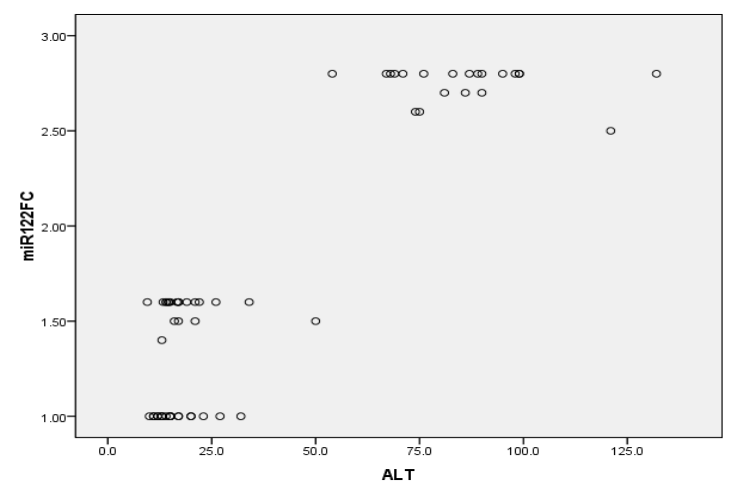

Fig. 1. Scatter Plot of the 2 Variables Folds change of miR-122 and ALT.

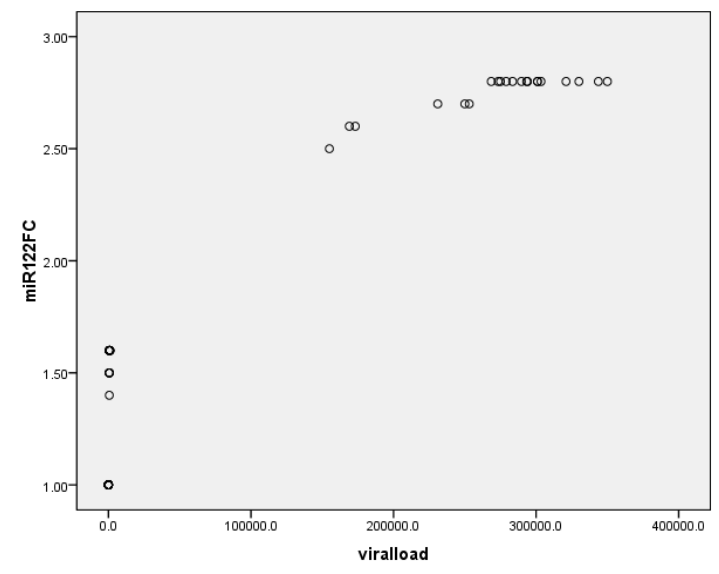

Fig. 2. Scatter Plot of the 2 Variables Folds Change of miR-122 and HBV Viral Load. 
searchers, the expression of miR-122 in serum, as a marker of tissue damage of liver, shows good specificity and sensitivity in detecting patients with chronic HBV infection (9-12). Zhou (2011) reported an increase in the level of serum miR-122 level in patients with liver cancer and chronic HBV infection (8). According to this researcher, measuring the miR-122 expression levels can serve as a biomarker and an indicator of the disease progression from $\mathrm{CHB}$ to $\mathrm{HBV}$ and $\mathrm{HCC}$.

Predicting the risk of disease progression in CHB patients plays an important role. A direct relation has been reported between the level of miR-122 and HBs antigen, which is an indicator of viral translation. In this regard, miR-122 levels can be an important indicator for progressing stages of the disease (9). Based on the obtained results, the fold change of miR-122 between each pair of groups was significantly different. Of course, the results of previous works revealed test group has an important role in the miR-122 expression level. For instance, Jian Zhou et al (6) reported a decrease on miR-122 expression levels in patients with hepatitis B with HCC compared with patients with $\mathrm{CHB}$.

Similar to the findings of this study, in a previous study in rodents, it was reported that liver injury induced by chemicals or alcohol leads to the enhanced level of plasma miR-122. This increase occurs before the increase in ALT, which is widely used as liver function marker (10). Ding et al reported a correlation between the miR-122 profiles levels and serum ALT levels in acute hepatotoxicity in humans compared with HCC-associated liver damage by HBV (13). Also, Farid et al reported that in human liver transplants a miR-122 level was significantly increased before and during acute transplant rejection (14). In another study, serum miR-122 was detected as a potential biomarker for liver damage. Moreover, an excellent correlation was observed between plasma miR-122 and the necroinflammatory activity of $\operatorname{HBV}(11,12)$.

Xianliang Hou et al reported that miR122 is positively correlated with ALT level and HBV DNA in patients with $\mathrm{CHB}$ and asymptomatic HBsAg carriers. Xing et al extracted a Positive correlation between expression of (miR29 and miR-122) and HBV DNA in patients with chronic hepatitis (3). In line with this study, we identified a positive correlation between the expression of HBV DNA and miR-122 in patients with CHB.

\section{CONCLUSION}

Briefly, a statistically significant relationship was found between an increase in the expression level of miR-122 and the occurrence of liver damage in patients with CHB. The detection of miR-122 can provide useful information for assessing the association between liver injury and HBV infection. The results showed that ALT levels are unchanged, especially in asymptomatic patients. However, further studies and more samples are needed for this purpose.

\section{Acknowledgment}

This manuscript is a part of a PhD thesis done in the High Institute for Research and Education in Transfusion
Medicine. We would like to thank the financial support of the Institute and Golestan Blood Bank Center for providing the collected samples.

\section{Ethics Approval and Consent to Participate}

Participants signed a written consent to participate in the study before sampling. This study was approved by the Ethics Committee of High Institute for Research and Education in Transfusion Medicine (Number: IR.TMI.REC.1396.013).

\section{Conflict of Interests}

The authors declare that they have no competing interests.

\section{References}

1. Adil B, Fatih O, Volkan I, Bora B, Veysel E, Koray K, et al Hepatitis B Virus and Hepatitis D Virus Recurrence in Patients Undergoing Liver Transplantation for Hepatitis B Virus and Hepatitis B Virus Plus Hepatitis D Virus. Transplant Proc. 2016;48(6):211923.

2. Dakic Z, Duric P, Fabri M, O'May F. Validity of hepatitis B and hepatitis C case definitions. J Infect Public Health. 2019;12(4):516521.

3. Hou X, Liang Y, Chen J, Wei Y, Zeng P, Wang L, et al. Expression Profiling of Cellular MicroRNA in Asymptomatic HBsAg Carriers and Chronic Hepatitis B Patients. Biomed Res Int, 2017. 2017: p. 6484835

4. Sharifi Z. Natural history of chronic hepatitis B virus infection based on laboratory testing. Iran J Public Health. 2014;43(7):990-3.

5. Zhou J, Yu L, Gao X, Hu J, Wang J, Dai Z, et al. Plasma microRNA panel to diagnose hepatitis B virus-related hepatocellular carcinoma. J Clin Oncol. 2011;29(36):4781-8.

6. Maimone S, Caccamo G, Squadrito G, Alibrandi A, Saffioti F, Spinella R, et al. A combination of different diagnostic tools allows identification of inactive hepatitis B virus carriers at a single time point evaluation. Liver Int. 2017;37(3):362-368.

7. Murakami Y, Toyoda H, Tanahashi T, Tanaka J, Kumada T, Yoshioka $\mathrm{Y}$, et al. Comprehensive miRNA expression analysis in peripheral blood can diagnose liver disease. PLoS One. 2012;7(10):e48366.

8. Ebrahimifard M, Zandi M, Moradi A. Evaluation of miR-122 levels in chronic HBV and liver cirrhosis patients. Arch Med Lab Sci. 2016;2(3).

9. Hu J, Xu Y, Hao J, Wang S, Li C, Meng S. MiR-122 in hepatic function and liver diseases. Protein Cell. 2012;3(5):364-71.

10. Chen Y, Cheng G, Mahato RI. RNAi for treating hepatitis B viral infection. Pharma Res. 2008;25(1):72-86.

11. Hayes CN, Akamatsu S, Tsuge M, Miki D, Akiyama R, Abe H, et al. Hepatitis B virus-specific miRNAs and Argonaute2 play a role in the viral life cycle. PloS One. 2012;7(10):e47490..

12. Zhang H, Li QY, Guo ZZ, Guan Y, Du J, Lu YY, et al. Serum levels of microRNAs can specifically predict liver injury of chronic hepatitis B. World J Gastroenterol. 2012;18(37):5188-96..

13. Ding X, Ding J, Ning J, Yi F, Chen J, Zhao D, et al. Circulating microRNA-122 as a potential biomarker for liver injury. Mol Med Rep. 2012;5(6):1428-32.

14. Farid WR, Pan Q, van der Meer AJ, de Ruiter PE, Ramakrishnaiah $\mathrm{V}$, de Jonge $\mathrm{J}$, et al. Hepatocyte-derived microRNAs as serum biomarkers of hepatic injury and rejection after liver transplantation. Liver Transpl. 2012;18(3):290-7. 